\title{
Abdominopelvic tumor mass in adolescent - a case report
}

\author{
Irina Mihaela Ciomaga ${ }^{1,5}$, Madalina Mardare ${ }^{1,5}$, Stefana Cristina Dupa ${ }^{1,5}$, \\ Cristina Alexa ${ }^{2}$, Doina Mihaila ${ }^{3}$, Elena Tarca ${ }^{4,5}$, Nicolai Nistor ${ }^{1,5}$ \\ ${ }^{1}$ Pediatrics, "Sf. Maria" Children's Emergency Hospital, lasi, Romania \\ ${ }^{2}$ Radiology and Medical Imaging, "Sf. Maria" Children's Emergency Hospital, Iasi, Romania \\ ${ }^{3}$ Pathology, "Sf. Maria" Children's Emergency Hospital, Iasi, Romania \\ 4Pediatric Surgery, "Sf. Maria" Children's Emergency Hospital, lasi, Romania \\ "University of Medicine and Pharmacy "Grigore T. Popa", Iasi, Romania
}

\begin{abstract}
A palpable mass in the abdominopelvic region in a teenager has usually severe manifestation. If we deal with such a mass, it must be stated whether it is malignant and if it is associated with the compression of the neighboring organs. Such a mass is most often discovered when the volume of the abdomen is increased, appears poor general condition or digestive or urinary symptoms.

The authors present the case of a 16-year-old adolescent who was admitted for the lumbar back pain, functional impotence of the right inferior limb and a tumor mass in the right iliac fossa 5 months prior. Physical examination, laboratory tests, imaging and pathology exams have revealed the diagnosis of rhabdomyosarcoma, embryonal type. The lack of communication between the teenager and the family delayed hospital presentation when the rhabdomyosarcoma was already at the terminal stage with pulmonary and bone metastases, with negative prognosis.
\end{abstract}

Keywords: rhabdomyosarcoma, tumor, teenager, malignant

\section{INTRODUCTION}

Abdominopelvic masses in children can be the expression of various disorders depending on their age, sex, sites and structure. In adolescent patients, it can be gynecological or not, benign or malignant (1). Rhabdomyosarcoma is one of the malignant tumors that may have this location, as is the case we will present below.

Rhabdomyosarcoma (RMS) is a malignant tumor of soft tissues (muscle, blood vessels, adipose tissue) that develops from striated muscle cells $(2,3)$. It is one of the most common soft tissue sarcomas, most cases occurring in young children (4). It represents $4.5 \%$ of all pediatric cancers (5) and is the third most common extracranialsolid tumor in children and adolescents after neuroblastoma and Wilms tumor (6). Patients with rhabdomyosarcoma can present with an asymp- tomatic mass or with signs and symptoms that are associated with the primary tumor site and are related to mass effect or complications that are secondary to the tumor $(6,7)$.

The objective of paraclinical exploration is to determine the size and possible compression upon adjacent neural structures and the presence of eventual metastases. This can be accomplished by ultrasound, CT or MRI.

The diagnosis should be confirmed histopathologically by the positive muscle markers in immunohistochemistry. The translocation $t(2,13)$ allows the diagnosis of alveolar forms. However biopsy is used to identify the relevant cells to rhabdomyosarcoma for histological variant and to provide clinical management and appropriate treatment strategies (3). Currently, multimodality treatment of rhabdomyosarcoma includes chemotherapy, surgery,radiotherapy with 
the risks associated with long-term treatment complications. Although the prognosis has improved a lot, for cases with metastases and relapses itcontinue to remain guarded (4).

\section{CASE PRESENTATION}

A 17-year-old patient with a 2 years history of a left ovarian cyst (for which she didn't do a check up), is admitted to "Sf. Maria" Children's Emergency Hospital Iasi for lumbar pain and functional impotence in the lower right limb, and the appearance of a mass in the right iliac fossa, a symptomatology that started insidiously about 5 months ago. The patient said that she performed the last medical check up almost two years ago. From the family history, we note that both the mother and an aunt are known to have ovarian polycystic disease.

On physical examination on admission, the patient was in moderate distress, no fever, normoponderal $\left(\mathrm{BMI}=19.94 \mathrm{~kg} / \mathrm{m}^{2}\right)$, warm, elastic, pale skin, marbled in the lower right leg, nonpalpable superficial ganglia. Examination of the osteo-articular system revealed pain and functional impotence in the right lower limb, and lumbar pain. She had normal vital signs (heart rate $=70 \mathrm{bpm}$ ) and pulsatile peripheral arteries. It was observed the presence of a mass at the level of the hypogastrium and right iliac fossa, imprecisely delimited, of tough consistency, adherent to the underlying tissues, tenderness to palpation. Normal transit and polakiuria. The patient is oriented to time and space, without signs of meningeal irritation.

Laboratory test: a complete blood count (CBC) revealed leukopenia $\left(2,730 \mathrm{GA} / \mathrm{mm}^{3}\right)$, mild thrombocytopenia $\left(110,000 / \mathrm{mm}^{3}\right)$ and mild anisocytosis (peripheral blood smear). Blood chemistry tests revealed hypophosphatemia (3.23 mg/dl serum phosphorus), increased LDH $(853 \mu \mathrm{l} / \mathrm{l})$, low ionic calcium $(3.97 \mathrm{mg} /$ $\mathrm{dl})$, increased ferritin $(151.3 \mu \mathrm{g} / \mathrm{dl})$. Coagulation tests, mandelic acid vanilloid, serum complement levels and amylazuria were normal, tests for syphilis, hepatitis $\mathrm{C}$ virus, hepatitis B virus and anti-Toxoplasma gondii (IgM) antibodies were negative. The microscopic examination of the peritoneal fluid revealed rare PMNs and red blood cells, the bacterioscopy being negative. Ovarian cancer was excluded by the normal value of the CA 125 antibody and the anti-Echinococcus ganulosus antibodies ganulosus were negative.

Posteroanterior chest $\mathbf{x}$-ray revealed multiple homogeneous, well-defined opacities of high intensity and variable sizes $(0.4-4 \mathrm{~cm})$, primarily located in the middle right lobe and left lower lobe.

The abdominal ultrasound revealed on the right lateral wall of bladder, possibly on the right ovary to- pography, a tumor with a non-homogeneous ecostructure (solid with internal transsonic areas), well circumscribed with a vascular signal and dimensions of $6.63 / 11.12 \mathrm{~cm}$, recommending pelvic IRM.

Multiple round-shaped, well defined, slightly nonhomogeneous nodules with liquid-type densities on unenhanced CT scan and contrast medium uptake on constrast - enhanced CT scan, which are distributed both lung parenchyma and have variable dimensions (the bigger has $36 / 36 \mathrm{~mm}$ size, and it is localised para-cardiac on the right (Fig. 1), in contact with the right inferior pulmonary vein and the pericardium. Multiple osteolytic lesions, circumscribed, located at the level of left-right VIII-IX arctic springs (Fig. 2), T2, L3 vertebral bodies and probably in the sternum (near the junction of the manubrious body).

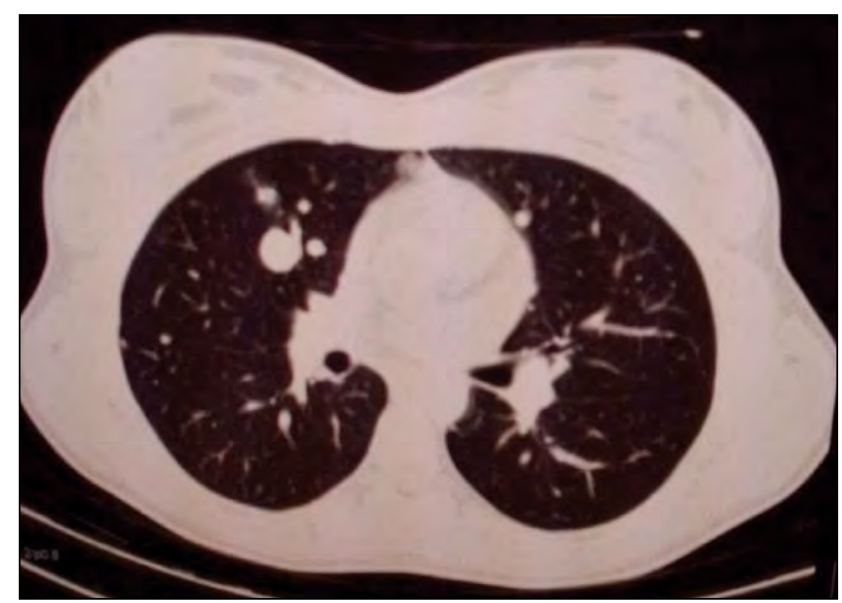

FIGURE 1. Thoracic cross section. Paracardial and pulmonary metastases

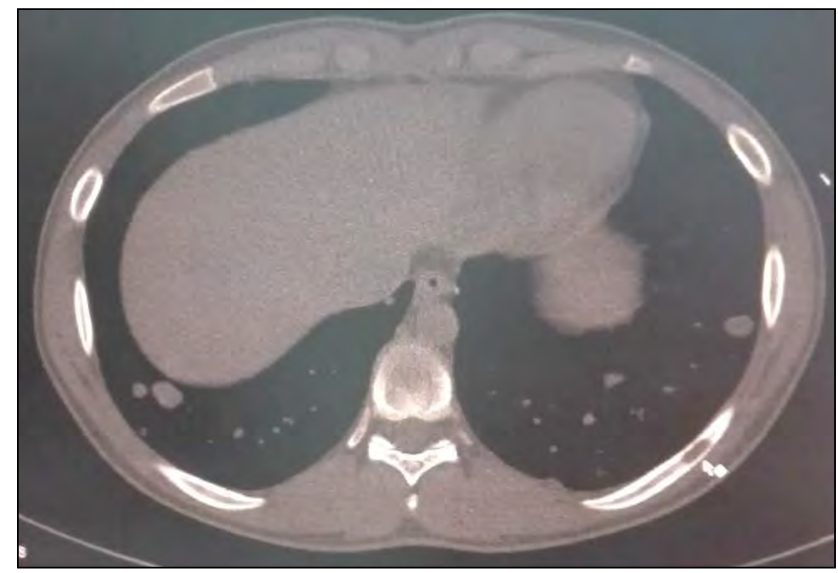

FIGURE 2. Thoracic cross section. Costal metastases

The MRI highlights a large, heterogeneous tumor, bounded by a fine capsule, mixed fluid, fat and solid signal (Fig. 4), which appears to be in the external and internal obturator muscles on the right side, with the secondary invasion upon adjacent structures (Fig. 3).

Secondary bone lesions in the pelvic bone and left femur (as evidenced by the MRI). Infiltrative aspect 
of the anal lift muscle, external genitalia and around the vaginal canal. Uterus and ovaries long ascended by the tumor.

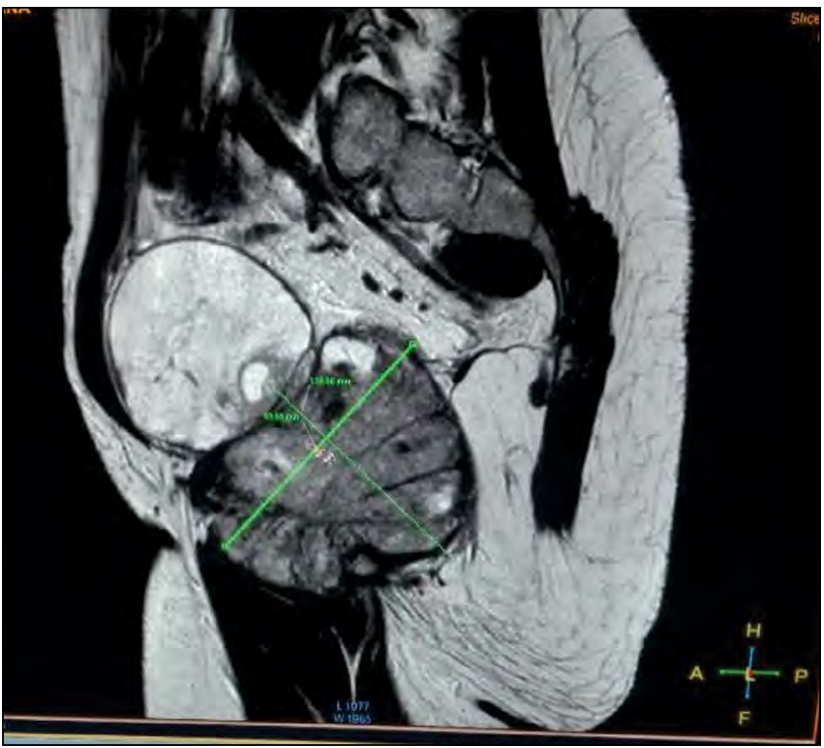

FIGURE 3. Sagittal abdominal-pelvic section. Tumor size 87/111/138 mm (antero-posterior, transverse / cranio-caudal)

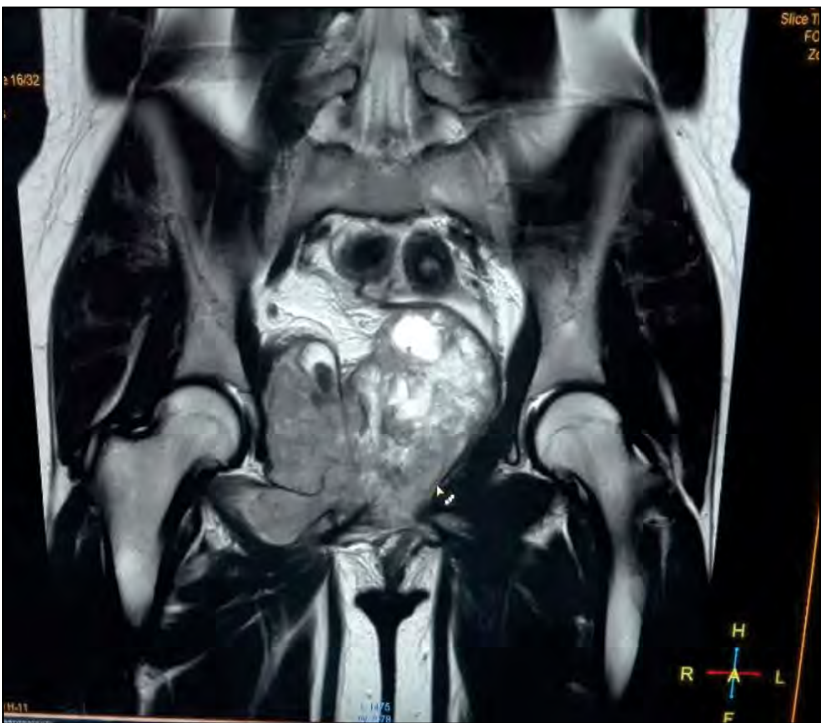

FIGURE 4. Abdominal-pelvic front section. We can observe the heterogeneous content of the tumor, fluid, fat and solid

\section{HISTOPATHOLOGICAL EXAMINATION}

The extracted fragments capture extensive areas of tumor necrosis and outbreaks of viable tumor cells in the periphery - embryonal rhabdomyosarcoma cell variant.

Immunohistochemistry - High Positive Vimentin (Fig. 7,8) and positive myogenin in tumor cells (Fig. 9), Ki67 > 10 positive cells in High Power Field $x 400$ (Fig. 10), CD34 negative in tumor proliferation (Fig. 11) and negative SMA in tumor cells (Fig. 12).

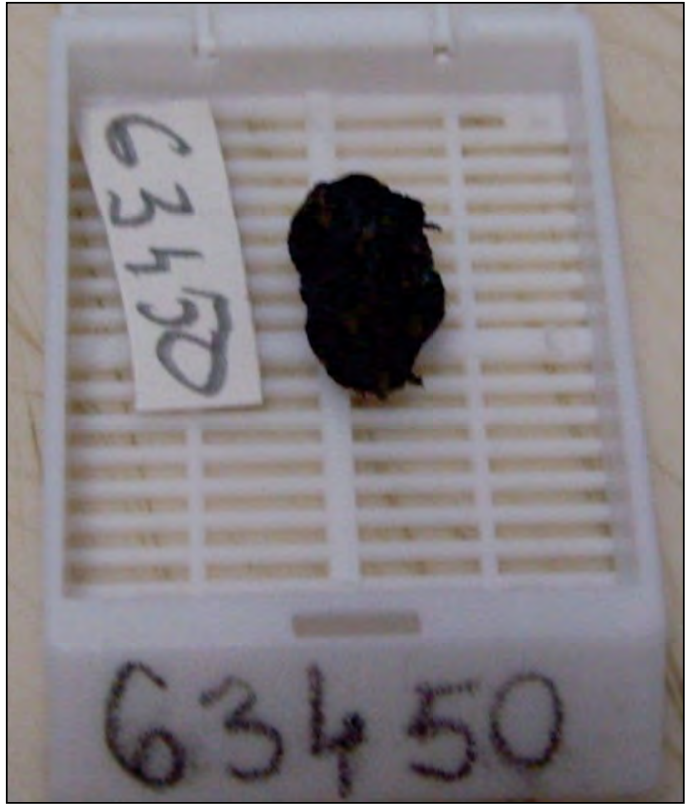

FIGURE 5. Biopsy - macroscopic aspect

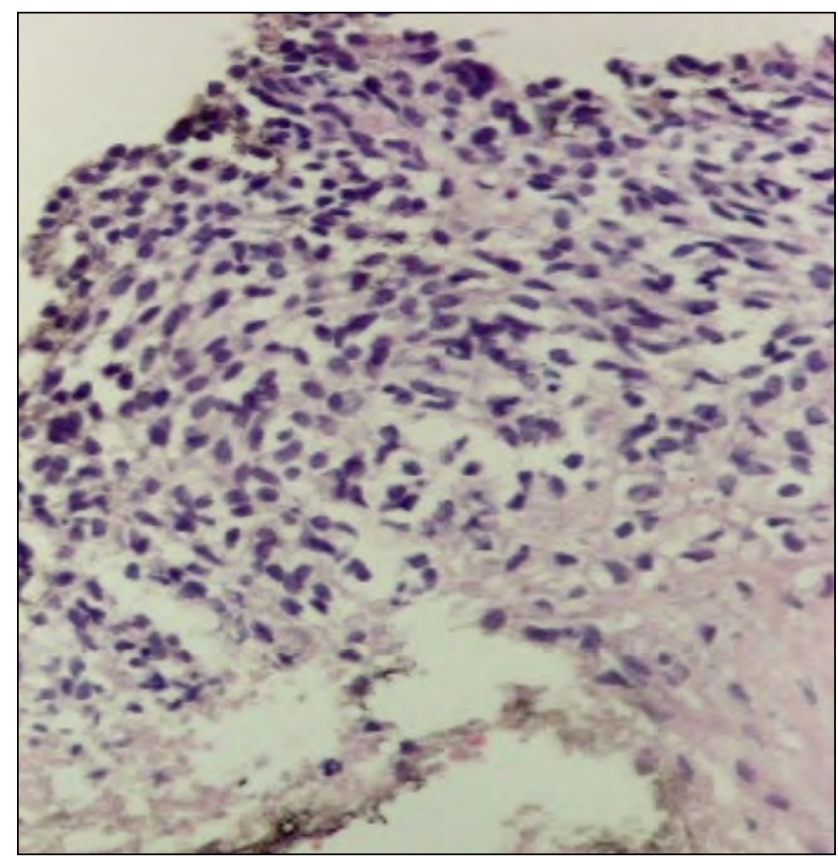

FIGURE 6. Microscopic aspect - HE X200

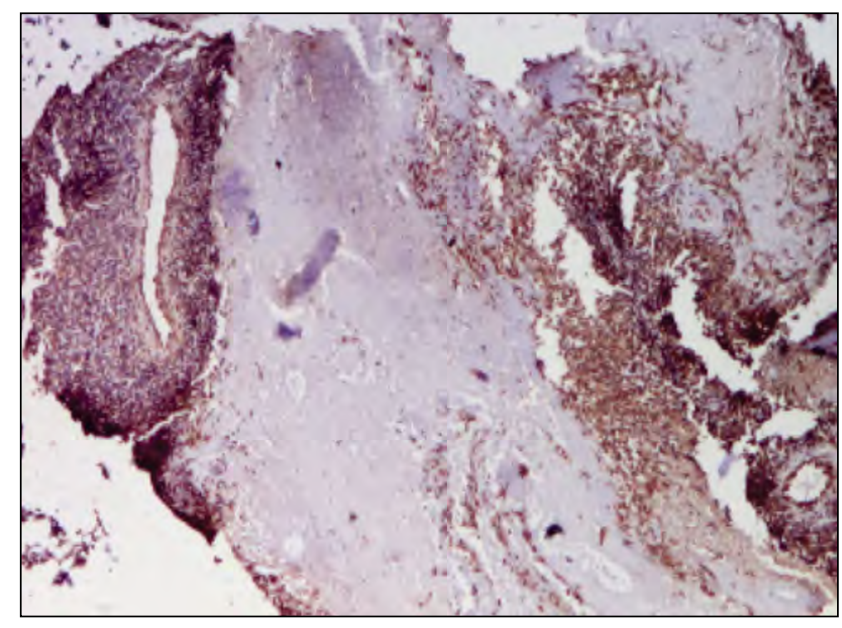

FIGURE 7. Vimentin $+X 40$ 


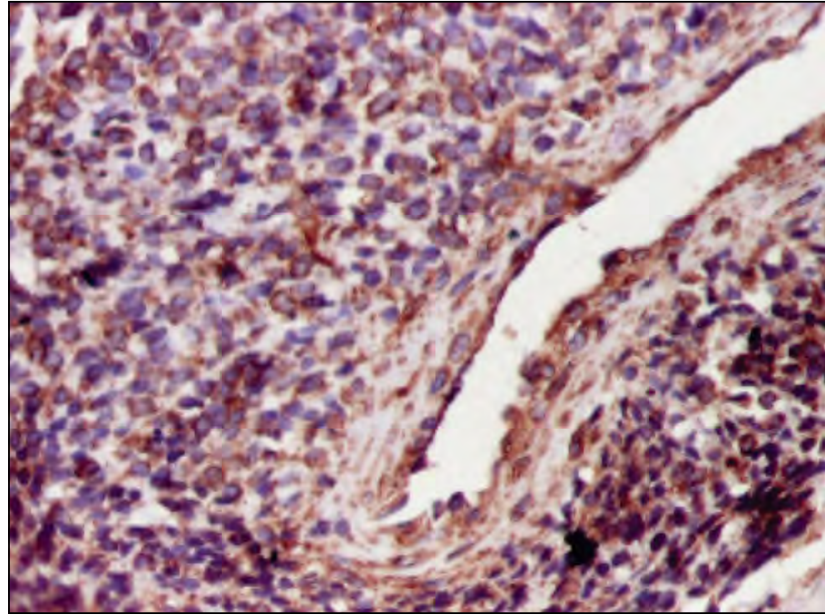

FIGURE 8. Vimentin $+X 200$

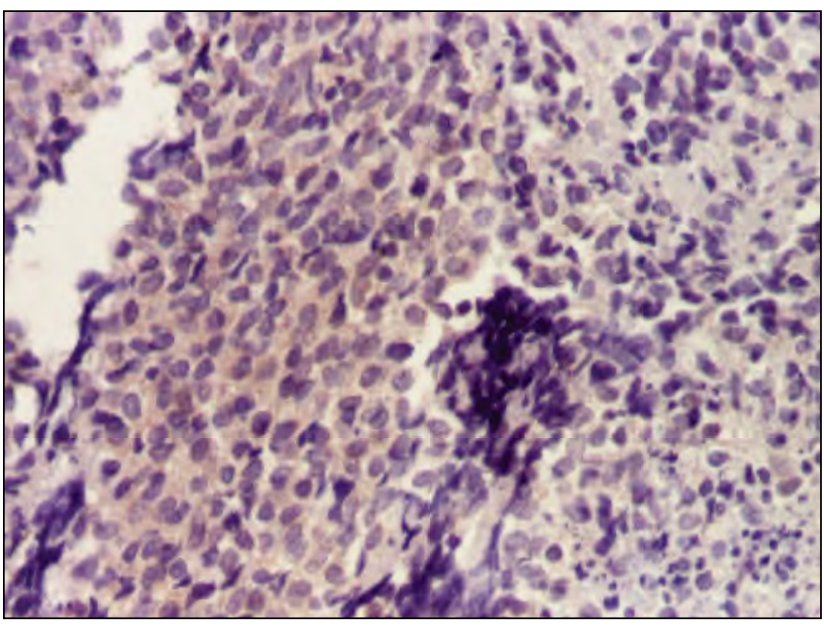

FIGURE 9. Myogenin $+X 200$

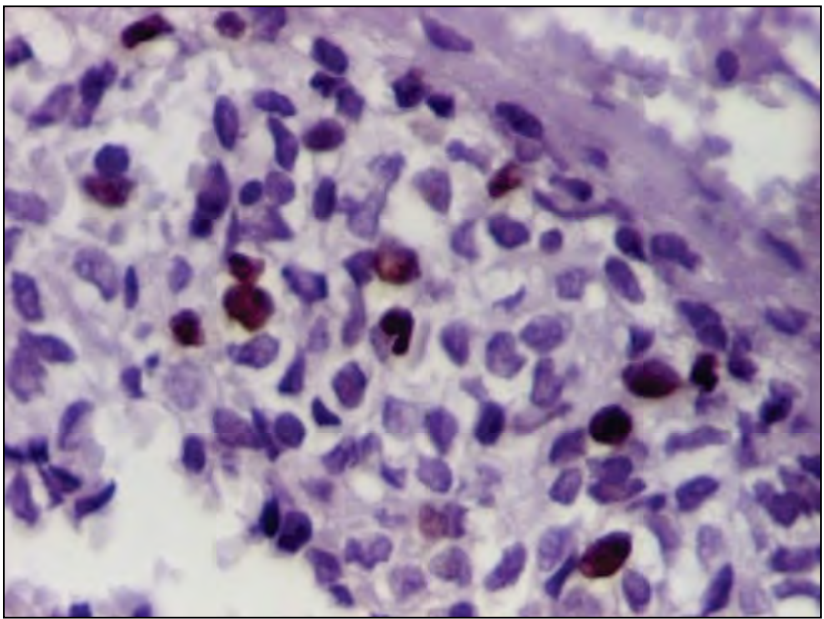

FIGURE 10. Ki67 $\times 400$

CT and MRI results with histopathological examination concluded the final diagnosis was stage IV rhabdomyosarcoma embryonal type.

\section{DISCUSSION}

Accidental detection of an abdominopelvic mass in a child or adolescent requires a precise and quick

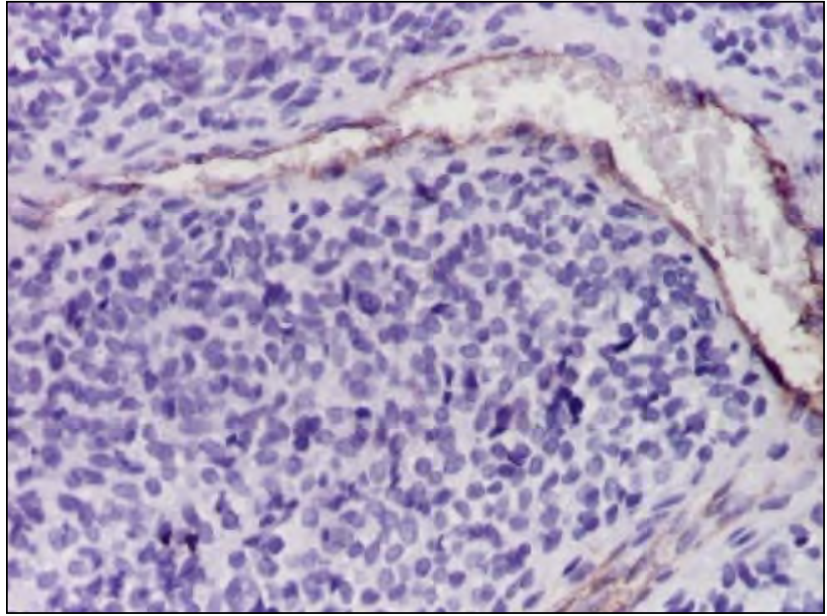

FIGURE 11. CD34 - X 200

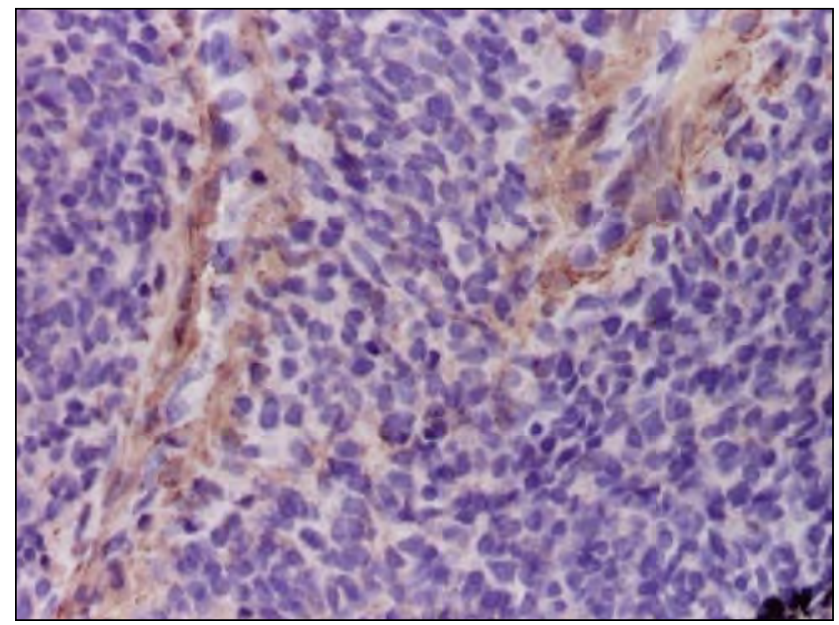

FIGURE 12. SMA - X200

diagnosis, because it is often the suggestive clinical sign of a malignant solid tumor. Taking an ultrasound avoids diagnosis traps by usually indicating the origin of the tumor and the site: retroperitoneal (renal or extrarenal), intraperitoneal or abdominopelvic, suggesting further investigations it may be needed (8). This adolescent patient was admitted for such an abdominopelvic mass, but she came late, about 5 months after apparent onset. Investigations revealed the diagnosis of rhabdomyosarcoma that was probably in the external and internal obturator muscles, with secondary invasion upon adjacent structures.

Rhabdomyosarcoma (RMS) is the most common soft tissue sarcoma (9). It is estimated that 350 new cases of RMS are diagnosed each year in patients under 20 years in the United States. In contrast, RMS is extremely rare in adults (6). There have also been reported congenital rhabdomyosarcomas cases with various sites $(10,11,12)$ as well as in the age of infants (cervix rhabdomyosarcoma at the age of 7 months) (13). Most rhabdomyosarcomas are sporadic, but some are associated with genetic syndromes: Li-Fraumeni syndrome, Rubenstein Taybi, Wiedemann- 
Beckwith, Costello, Noonan and type I neurofibromatosis (6). Rabdomiosarcomas with pelvic site are rare. A large study, including children and adults, reported a frequency of pelvic sites at $3.3 \%$ (14). As RMS is derived from primitive mesenchymal stem cells directed towards myogenesis, it can arise in a variety of anatomic sites throughout the body (15). According to the WHO classification, histological types of RMS include embryonal RMS with its variants: botrioid and anaplastic, alveolar RMS, with its solid variant and mixed embryonal-alveolar variant and pleomorphic RMS. Spindle cell rhabdomyosarcoma is no longer considered as a variant of embryonal RMS but a separate entity with NCOA2 gene rearrangements (16). This case report was a rhabdomyosarcoma embryonal variant. Incidentally, the embryonal type is by far the most common in the children compared to the alveolar type. A study of 25 children with rhabdomyosarcoma previously performed at the same hospital found in 20 cases the embryo type (17). A larger study of 277 patients of all ages reported an $87.4 \%$ embryotype, $9.4 \%$ alveolar and pleomorphic type in $1.8 \%$ of cases (14).Currently, multimodality treatment that includes chemotherapy, surgery with or without radiation therapy, had become the standard care for RMS (6). Survival rates for adolescents and adults with RMS, on the other hand, are significantly worse compared to younger children (range 21\%-56\%) $(18,19)$. One of the prognostic factors is age: children aged 1 to 9 years have favorable prognostic, while those younger than 1 year and older than 9 years have worse prognostic (5-year survival is $76 \%$ for patients $<1$

\section{REFERENCES}

1. Francis JC, Dietrich JE. You have identified a pelvic mass in your teenage patient. What now? OBG Management 2015 ; 27 (7): 21-26.

2. Dasgupta R, Rodeberg DA, Update of rhabdomyosarcoma. Semin Pediatr Surg 2012; 21 (1): 68-78.

3. Cadas C. Prise en charge d'un patient atteint de rhabdomyosarcome. HAL archives-ouvertes 2017. HAL Id: dumas-01546119.

4. Wasti AT, Mandeville H, Gatz S, Chisholm JC. Rhabdomyosarcoma. Pediatrics and Child Health 2018; 28 ( 4): 157-163.

5. Reilly BK, Kim A, Peña MT, Dong TA, Rossi C, Murnick JG et al. Rhabdomyosarcoma of the head and neck in children: review and update. Int J Pediatr Otorhinolaryngol. 2015; 79: 1477-1483.

6. Egas-Bejar D, Huh WW. Rhabdomyosarcoma in adolescent and young adult patients: current perspectives. Adolescent Health, Medicine and Therapeutics 2014:5 115-125.

7. Bloem L, I. Reidsma I. Bone and soft tissue tumors of hip and pelvis. European Journal of Radiology 2012; 81: 3793-3801.

8. Orbach D, Gajdos V, Doz F, André N. Pièges et urgences diagnostiques des cancers de l'enfant. La Revue du Praticien 2014; 64: 1276-1283.

9. Yang L,Takimoto T, Fujimoto J. Prognostic model for predicting overall survival in children and adolescents with rhabdomyosarcoma. BMC Cancer 2014, 14:654.

10. Eghtedari M, Farsiani A.R, Bordbar M.R. Congenital Orbital Rhabdomyosarcoma. Ocul Oncol Pathol 2018; 4:165-169. year, $87 \%$ for patients $1-9$ years, and $76 \%$ for patients $>10$ years $(20,21)$. Other factors that affect prognosis are: primary tumor site, the size of tumor, resectability, presence or absence of metastases, number of metastatic sites, lymph node status, histopatholgic subtype (alveolar vs. embrional), and, in some cases, delivery of radiation therapy $(3,22,23)$. The patient was discharged against medical advice although she was informed about prognosis and survival rate, refusing conventional treatment.

\section{CONCLUSIONS}

The presence of a rhabdomyosarcoma with abdominopelvic site in a teenager is unusual. It should always be considered in the differential diagnosis in a child with an abdominal-pelvic mass that can not be precisely defined as belonging to a certain organ, knowing that rhabdomyosarcoma can occur in any organ or tissue except bones. In this case report the diagnosis was delayed when pulmonary and bone metastases were already in place and the prognosis was guarded. The lack of cooperation between the adolescent patient and her family who have refused any medical treatment aprroach has further reduced the chances of survival and better outcome.

The patient's family decided (the patient being a minor) to refuse the proposed treatment and requested the discharge, the doctors being forced to comply with this decision. Despite their efforts to follow alternative therapies, the outcome was the same, extending the survival by one year.

11. Esmaeili H, Mahzad Azimpouran M. Congenital embryonal rhabdomyosarcoma; multiple lesions. International Journal of Surgery Case Reports 2017;31: 47-50.

12. Taeubner J, Brozou T, Qin N, Barti J, Ginzel S, Schaper J, Felsberg J, Fuldas C, Vokohl C, Borkhardt A, Kuhlen M. Congenital embryonal rhabdomyosarcoma caused by heterozygous concomitant PTCH1 and PTCH2 germline mutations. European Journal of Human Genetics 2018; 26:137-142.

13. Yasmin F, Ahmed MAU, Begum T, Ahmed T, Baki MA. A Case Report of Rhabdomyosarcoma of Uterine Cervix in a 7-Month-Old Child. BIRDEM Med J 2017; 7(3): 242-244.

14. Ahmad Z, Din NU, Ahmad A, Imran S, Pervez S, Ahmed R, Kayani N. Rhabdomyosarcoma - an Epidemiological and Histopathologic Study of 277 Cases from a Major Tertiary Care Center in Karachi, Pakistan. Asian Pacific Journal of Cancer Prevention, Vol 16, 2015; 16: 757-770.

15. Soleimani VD, Rudnicki MA. New insights into the origin and the genetic basis of rhabdomyosarcomas. Cancer Cell 2011; 19 (2): 157-159.

16. Vy J, Fletcher CDM . WHO classification of soft tissue tumours: An update based on the 2013 (4th) edition. Pathology 2014; 46 (2):95-104.

17. Diaconescu S, Burlea M, Miron I, Aprodu SG, Mihaila D, Olaru C, Miron L. Childhood rhabdomyosarcoma. Anatomo-clinical and 
therapeutic study on 25 cases. Surgical implications. Rom J Morphol Embryol 2013; 54 (3): 531-537.

18. Bisogo G, Compostella A, Ferrari A, Pastore G,Cecchetto G, Garaventa A, Indolfi P. Rhabdomyosarcoma in adolescents: A report from the AIEOP Soft Tissue Sarcoma Committee. Cancer 2012 Feb 1; 118(3):821-7.

19. van Gaal JC, van der Graaf WTA, Rikhof B et al. Age as a prognostic factor for outcome in rhabdomyosarcoma (RMS) patients (pts). In: ASCO annual meeting 2008.

20. Malempati S, Hawkins DS. Rhabdomyosarcoma: Review of the Children's Oncology Group (COG) Soft-Tissue Sarcoma Committee experience and rationale for current COG studies 2012; 59 (1):5-10.
21. Domenichelli V, Straziuso S, Italiano F, Pelusi G, Federici S. Rhabdomyosarcoma (RMS) of the Renal Pelvis in a Child: A Case Report. Urol Androl Open J. 2017; 1(2): 35-38.

22. Levy CF, Wexler LH. Rhabdomyosarcoma and Other Soft-Tissue Sarcomas. In: Lanzkowsky P, Lipton JM, Fish JD (eds). Lanzkowsky's Manual of Pediatric Hematology and Oncology. Academic Press 2016; 505-523.

23. Kim JR, Yoon HM, Koh KN, Jung AY. Rhabdomyosarcoma in Children and Adolescents: Patterns and Risk Factors of Distant Metastasis. American Journal of Roentgenology. 2017;209: 409-416.

Conflict of interest: none declared Financial support: none declared 